\title{
A unifying force
}

\author{
The questions to be explored at the Large Hadron Collider offer a chance to rekindle public interest \\ in the fundamental principles of the Universe in which we live.
}

Tour he Large Hadron Collider (LHC) that is nearing completion outside Geneva, Switzerland, is a testament to two of the greatest human qualities: a fascination with the workings of the Universe and an ability to cooperate to achieve shared goals. After the machine - now the centrepiece of Europe's particle-physics laboratory, CERN - goes into operation next year, it should allow experimentalists for the first time in decades to blaze paths into areas on which settled theory stands silent (see page 269).

The physicists exploring this new world will do so as a unified global community. Although CERN is broadly a European achievement, in which the continent can take great pride, the LHC and its attendant detectors have received contributions in cash or kind from more or less every country with the capability to participate in this sort of frontier science. The LHC therefore sets a new high-water mark in disinterested global cooperation.

But it is important to distinguish disinterested from uninterested. The nations of the world are not investing in the LHC because they necessarily expect to see returns similar to those physics delivered in the twentieth century (most obviously and terribly in the realm of nuclear weapons). At the same time, it is becoming apparent that such investments in physics no longer elicit quite the thrill that they once did among the general public.

Interest in the advances made at the LHC's immediate predecessors - the Large Electron Positron Collider at CERN and the Tevatron at America's Fermilab - drops off fairly steeply as one leaves the precincts of high-energy physics. Although the public likes the idea that scientists are making fundamental progress, the advances made in particle physics can seem increasingly far removed from ideas that resonate in the common imagination. In the first half of the twentieth century, the nucleus, relativity, the quantum and the uncertainty principle were quickly imbued with cultural meaning far beyond their scientific context. Gauge symmetries and the Higgs boson have yet to acquire such broader, symbolic importance.

Many physicists will consider it fanciful to suggest that they should - not least because the meanings that became associated with such concepts outside the realms of physics have often been far removed from, or in direct contradiction of, their scientific meaning.

At the same time, it is hard to ask people to spend the large sums needed to explore the frontiers of particle physics if they do not have some sense of investment in the questions that it asks. Here, the LHC offers an opportunity to re-establish a resonance between particle physics and the broader culture in which it sits. There is a strong case that the Universe is made up in large part of 'dark' matter and energy, quite unlike constituents that we can observe directly (see pages 240 and 245). It is possible that observations made by the LHC's detectors will
"Advances made in particle physics can seem increasingly far removed from ideas that resonate in the common imagination." speak directly to the nature of these, perhaps even producing in the laboratory some of the dark matter that seems to dominate the spiralling and clustering of galaxies.

The discovery of the hidden constituents of the Universe is a grand task, and has an imaginative appeal worthy of the global effort under way at CERN. And an ever stronger bond between the study of fundamental forces and particles on the one hand, and the structure and history of the Universe on the other, bodes well for the future of particle physics in other ways, too. Magnificent though machines such as the LHC are, they will necessarily be few and far between. It is important to have alternative ways forward that are less resource-intensive. Particle-flavoured astrophysics offers many opportunities along these lines, as do small-scale experiments looking for phenomena such as neutrinoless double-beta decay (see page 232).

CERN's grandeur comes in part from the paired missions of unification that it is embarked on - a theoretical unification of the phenomena of physics and a practical unification of the scientific aspirations of the world. Unifying the very large with the very small adds to the excitement generated, and should unite the imaginations of the world, helping to restore the prized position our culture has reserved for fundamental physics.

\section{Transmission lines}

\section{Field trials of AIDS prevention methods are as essential as they are politically awkward.}

M ore people than ever have access to effective AIDS treatments. But the virus will never be contained without more effective measures to prevent transmission - and the need for measures that can be initiated by women is especially urgent.

Unfortunately, the run-up to this year's International AIDS Society
(IAS) conference in Sydney, Australia, has been dominated by negative research results concerning female-initiated prevention. But scientists and advocates should keep working resolutely together to make sure that testing of such methods continues apace.

The vast majority of new HIV infections in Africa, where the pandemic is most severe, occur through heterosexual transmission. But women are often powerless to negotiate the use of condoms - by far the best way to prevent infection. This is the impetus behind the clinical trials now testing alternative female-initiated prevention techniques. These include microbicides - gels or creams applied to the vagina to block infection; barrier methods, such as diaphragms; 